\title{
Methane sensing using multiple-coupling gaps in hollow-core photonic bandgap fibers
}

\author{
J. M. Lazaro*a , A. M. Cubillas ${ }^{a}$, M. Silva-Lopez ${ }^{a}$, O. M. Conde ${ }^{a}$, M. Petrovich ${ }^{b}$ and J. M. Lopez-Higuera ${ }^{a}$ \\ ${ }^{\mathrm{a}}$ Grupo de Ingeniería Fotónica, Dpto. TEISA, Universidad de Cantabria, Avda. Los Castros S/N, \\ C.P. 39005 Santander, Spain; \\ ${ }^{\mathrm{b}}$ Optoelectronics Research Centre, University of Southampton, Southampton SO17 1BJ, UK \\ *lazarojm@unican.es; phone 34942 200877- Ext 18; fax 34942 200877; http://gif.teisa.unican.es/
}

\begin{abstract}
Gas detection and gas sensing based on hollow core photonic bandgap fiber (HC-PBF) is a very promising technique due to the long interaction light-gas lengths that are achievable. However, long path-lengths also imply higher gas filling times of the hollow fiber and higher response times of the detection systems what can constitute a serious practical inconvenience. In this paper, the high sensitivity is maintained but the sensor response time is reduced by using multiplecoupling fiber gaps. The results and conclusions extracted from a systematic experimental study (comparing the spectra and filling time of different HC-PBF lengths and different number of coupling gaps) are presented and discussed. Finally, the maximum number of gaps allowed in the system is modelled.
\end{abstract}

Keywords: Hollow-core photonic bandgap fibers, Multi-coupling gaps, Near-infrared Spectroscopy, Gas sensing, Methane.

\section{INTRODUCTION}

Gas monitoring is essential in many industrial, environmental and biomedical applications. Methane detection, in particular, is extremely important for safety and security reasons since it is an inflammable gas, with a lower explosive limit (LEL) of 5\% methane in air. As a consequence, it is necessary to develop methane sensors to ensure that its concentration is below this safety limit.

Optical sensors are an attractive alternative for gas detection due to its high spectral resolution, precise gas species identification and possibility of remote measurements [1]. Photonic crystal fibers (PCF) [2] provide new properties that make them suitable for sensing applications [3-6]. Specifically, hollow core photonic bandgap fibers (HC-PBF) [7] are very appropriate for gas sensing applications due to the high overlap achieved between the light and the gas confined in its core [3]. It has already been demonstrated the detection of methane concentration below the LEL using HC-PBFs $[5,6]$. Therefore, weak absorption gases can be monitored with this technology because longer iteration lengths can be obtained. At the same time, sensors employing this technology become compact as the fiber can be coiled up. As the Beer-Lambert law states, longer interaction lengths give rise to higher absorption rates $[1,8]$. However, the use of longer pathlengths with HC-PBFs yields to an increase of the diffusion time of the gas through the core of the fiber, thus increasing the response time of the sensor.

In order to reduce the response time of the sensor is therefore necessary to ensure fast gas diffusion inside the hollowcore. Several configurations can be envisaged to provide multiple access points for the gas to reach the hollow-core through the cladding. However, methods such as hole drilling produce an asymmetrical transverse disruption of the periodic cladding structure and require expensive materials to make the access points. Consequently, the filling process becomes more complex [9-11]. Multi-coupling gaps have been proposed as another alternative, being a very promising technique for a practical sensor implementation $[12,13]$. However, sensors using this method have not been realized yet. In this paper, we demonstrate the application of a HC-PBF with multi-coupling gaps to the detection of methane.

\section{EXPERIMENTAL SETUP}

The gas in-diffusion into the hollow fiber was experimentally monitored measuring the spectral response evolution of different fiber lengths and different number of coupling gaps during the process. Then filling time for each case has been evaluated. The 1300-nm band of methane $\left(v_{2}+2 v_{3}\right)$ has been chosen for the experiments. Although the 1300-nm band of methane is a weak absorption band, it corresponds to one of the telecommunication bands where the low attenuation of 
standard optical fibers allows the possibility of remote detection. Furthermore, this band is attractive because of the availability of cheap light sources and detectors.

The HC-PBF used in the experiment was supplied by the Optoelectronics Research Centre from Southampton, and it is shown as an inset in Figure 1. The fiber core diameter is $10 \mu \mathrm{m}$, which corresponds to seven missing unit cells from the holey region. The un-buffered overall fiber diameter is $185 \mu \mathrm{m}$. The normalized transmission of the HC-PBF is shown in Figure 1. The fiber has a bandgap guidance of around $1220-1380 \mathrm{~nm}$, with an estimated loss of $0.2 \mathrm{~dB} \cdot \mathrm{m}^{-1}$ in this wavelength range. This bandgap guidance makes this HC-PBF suitable for detecting methane at its $v_{2}+2 v_{3}$ band.

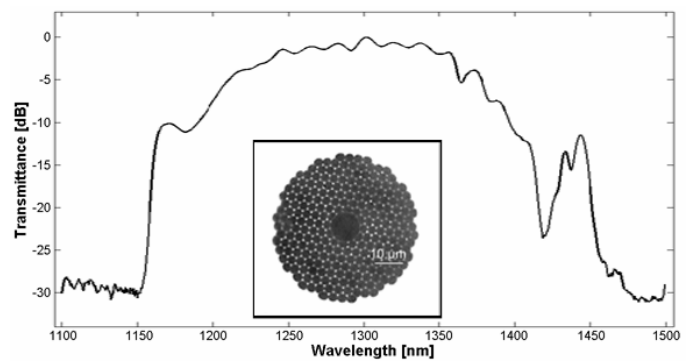

Fig. 1: Normalized transmission of the HC-PBF employed in the experiments. Inset: Microscope image of the HC-PBF cross section.

The fiber was interrogated with a LED source (Agilent 83437A) and the spectral response was recorded with an OSA (Agilent 86142A). The fiber was placed between these two devices with different configurations (see Figure 2). Firstly, measurements with the total length of the fiber $(4.9 \mathrm{~m})$ were realized as in configuration 1 . The fiber was then half divided in two sections and measurements were repeated for one length section of $2.45 \mathrm{~m}$ (configuration 1) and for two sections of $2.45 \mathrm{~m}$ joined using the multiple-coupling gaps method (configuration 2). In a next step, these two sections were again half divided obtaining four HC-PBF sections of $1.225 \mathrm{~m}$. In this case, measurements were repeated for a section (configuration 1) and for the four sections coupled using the multi-coupling method (configuration 3 ). In every configuration, each section has an open end and the other one is fused to a standard optical fibre (the splice attenuation was measured to be $1 \mathrm{~dB}$ ). In this way, we ensure the gas diffuses through the fibers in the same way. V-grooves were used to couple the light between the open ends of the fiber sections. The aligned sections of HC-PBF were placed in a gas chamber in which a methane concentration of $18750 \mathrm{ppm}(1.875 \%)$ in air was introduced at a relative pressure of 1 bar.

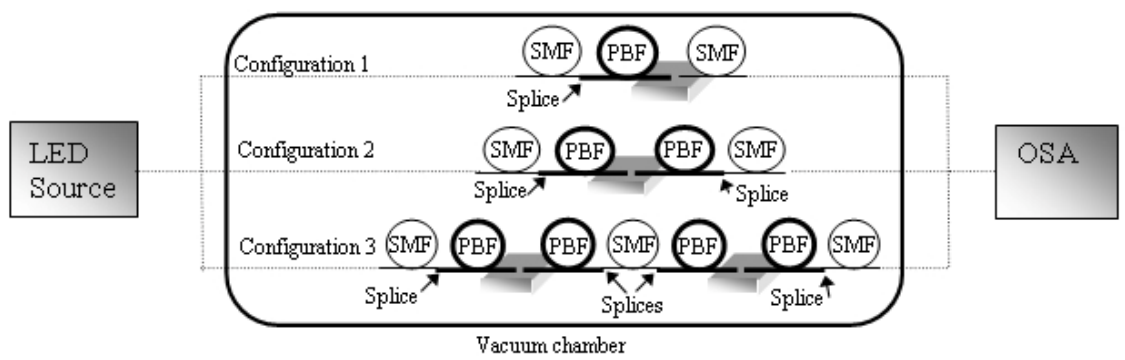

Fig. 2: Experimental setup for the different configurations. SMF: single-mode fiber; PBF: hollow core photonic bandgap fiber; OSA: optical spectrum analyser.

\section{RESULTS}

The comparison of the three different fiber lengths $(4.9 \mathrm{~m}, 2.45 \mathrm{~m}$ and $1.225 \mathrm{~m})$ for the one-section scheme (configuration 1 of Figure 2) was first evaluated. Figure 3(a) shows the measured spectra of methane in the 1300-nm band for the different HC-PBF lengths values. The stronger absorption line that can be seen in Figure 3(a) is the Qbranch, centered at $1331.55 \mathrm{~nm}$. Figure 3(b) shows the transmission at the Q-branch as a function of time for the different HC-PBF lengths during the diffusion of methane into the fiber. The behaviour has been fitted with a double exponential equation. The fitting provides the time constant of this process, which is defined as the time value in which the transmittance reaches an steady state. We have chosen the time value in which the derivate of the curve fit is 0.005 , i.e. the slope of the curve is almost zero. The obtained values for the time constant are 14.42, 5.68 and 2.87 minutes for the lengths of $4.9 \mathrm{~m}, 2.45 \mathrm{~m}$ and $1.225 \mathrm{~m}$, respectively. As expected, longer path-lengths increase the absorbance but also the time needed for the gas to diffuse into the fiber. 
(a)

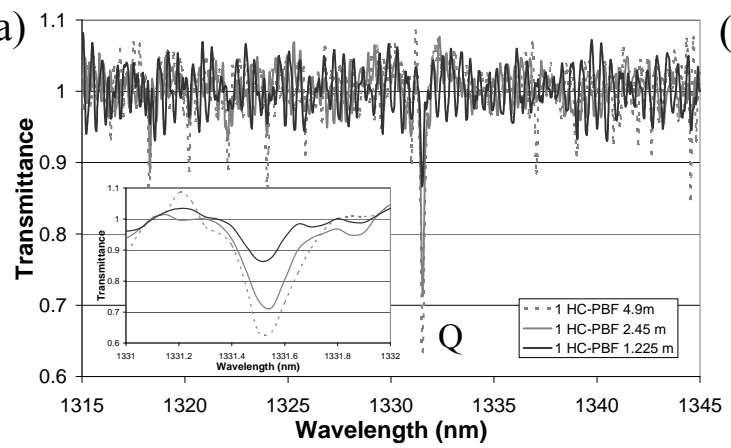

(b)

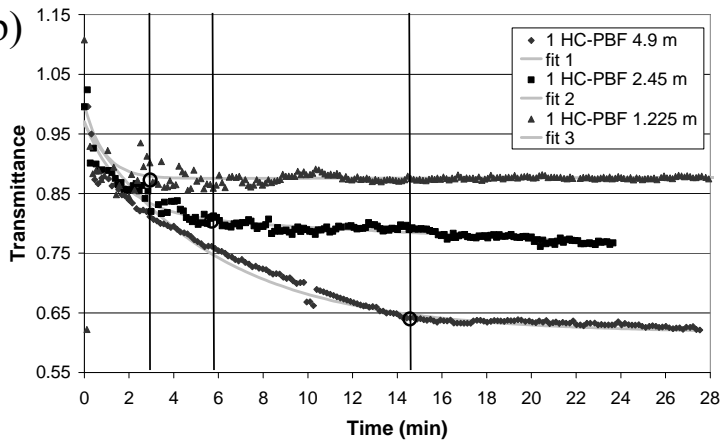

Fig. 3: Measured normalized transmittance at Q-branch: a) as a function of the HC-PBF length (inset: zoom of the Qbranch), b) as a function of time for different lengths while the methane is diffusing into the HC-PCF (dots) and double exponential fits of the diffusion time (solid lines)

The filling time for the multiple-coupling gaps technique was then evaluated. This corresponds to configurations 2 and 3 in Figure 2. Figure 4 shows the comparison of the methane absorption spectra and the filling times, in the cases in which the total length of the fibre is $4.9 \mathrm{~m}$ ( 1 section of $4.9 \mathrm{~m}, 2$ sections of $2.45 \mathrm{~m}$ and 4 sections of $1.225 \mathrm{~m})$. Although a small difference in the Q-branch transmittance of the spectrum can be observed (of around $0.05 \mathrm{~dB}$, see inset of Figure 4 (a)), this may be due to noise. However, the transmission spectra for the three lengths is very similar.
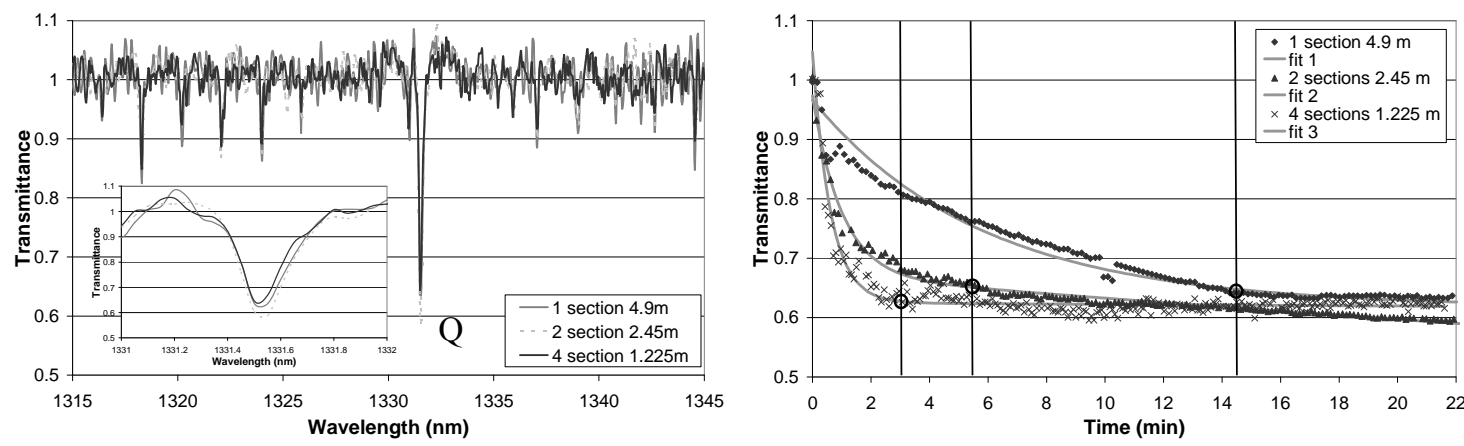

Fig. 4: Measured of total HC-PBF length of $4.9 \mathrm{~m}$. a) Transmission spectrum of methane measured at room temperature and a relative pressure of 1 bar with a methane concentration of $18750 \mathrm{ppm}$ in air. Q-branch is labelled (inset zoom of the Q-branch). b) Normalized transmittance at Q-branch as a function of time while the methane is diffusion into the HCPCF (dots) and double exponential fits of the diffusion time (solid lines).

In Figure 4(b), the measured values for the filling tine, as well as the double exponential fitting of the curves are shown. The time constant values, obtained using the same method described above, are 5.47 minutes for two sections of $2.45 \mathrm{~m}$ and $3.05 \mathrm{~min}$ for four sections of $1.225 \mathrm{~m}$. If we compare these values with the time constants obtained in the one-section scheme (Figure 3(b)), it can be observed that the diffusion time using multiple gaps corresponds to the time needed for the gas to diffuse into one sections of fiber. The results show that multiple-coupling gaps method substantially reduces the filling time while keeping the absorption value of the gas due to the total length of the HC-PBF. These results demonstrate the benefits of using HC-PBFs with multiple-coupling gaps for sensor applications.

Finally, an equation of the maximum number of gaps allowed in the system is obtained. We can generalized the equation proposed in reference 12 to the case of a remote sensing. In this way, including the losses due to the standard fibres and the splices, the following expression is obtained:

$$
P_{\text {out }}=\frac{P_{\text {in }}}{\left(\alpha_{\text {gas }}(C)+\alpha_{H C-P B F}\right) \cdot L_{H C-P B F}+n_{g} \cdot l_{g}+n_{s} \cdot l_{s}+\alpha_{S M F} \cdot L_{S M F}}
$$

Where $P_{\text {out }}$ and $P_{\text {in }}$ are the powers at the output and the input of the system, respectively. $\alpha_{\text {gas }}(C), \alpha_{\mathrm{HC}-\mathrm{PBF}}$ and $\alpha_{\mathrm{SMF}}$ are the attenuation coefficient as a function of the length due to the gas absorption, the HC-PBF and the SMFs, respectively. It is important to note that the gas attenuation coefficient depends on the gas concentration $(\mathrm{C})$. $\mathrm{L}_{\mathrm{HC}-\mathrm{PBF}}$ and $\mathrm{L}_{\mathrm{SMF}}$ are the 
lengths of the HC-PBF sensing fiber and SMF transmission fibers, respectively. Finally, $\mathrm{n}_{\mathrm{g}}, \mathrm{l}_{\mathrm{g}}, \mathrm{n}_{\mathrm{s}}$ and $\mathrm{l}_{\mathrm{s}}$ are number and the losses of gaps between HC-PBFs (subscript g) and splices between SMF and HC-PBFs (subscript s). The minimum power that can be detected is limited by the detector sensitivity $\left(\mathrm{S}_{\mathrm{D}}\right)$, therefore the maximum number of gaps allowed in the system can be obtained with the following expression:

$$
n_{g}=\frac{P_{i n}-\left\lfloor\left(\alpha_{\text {gas }}(C)+\alpha_{H C-P B F}\right) \cdot L_{H C-P B F}-n_{s} \cdot l_{s}-\alpha_{S M F} \cdot L_{S M F}\right\rfloor S_{D}}{l_{g} \cdot S_{D}}
$$

It is worth mentioning that any reduction in the system losses will increase the maximum number of gaps allowed, thus reducing the sensor response time. Furthermore, the attenuation coefficient of a standard telecommunication fiber is below $0.4 \mathrm{~dB} \cdot \mathrm{km}^{-1}$ for the $1300-\mathrm{nm}$ band. Therefore, remote sensing capability is also provided without reducing significantly the maximum number of gaps in the system.

\section{CONCLUSIONS}

We have demonstrated a methane sensor based on multi-coupling gaps in HC-PBFs. Methane band at 1300-nm was chosen as it is in the second window of communication and there are many sources and detectors available. Multicoupling gaps in HC-PBFs have been proved to reduce the filling time while maintaining the absorption of the gas. Finally, an expression of the maximum number of gaps has been proposed. It is shown that minimum losses are needed in the system to increase the maximum number of gaps allowed and, therefore, reduce the response time. It can be concluded that with this method a suitable sensor to measure methane can be realized due to its sensibility and response time.

\section{ACKNOWLEDGMENTS}

This work was supported in part by the Spanish Government TEC2004-05936-C02, TEC200508218-C02-02 and TEC2007-67987-CO2-01 projects

\section{REFERENCES}

J. M. López-Higuera, Editor. Handbook of Optical Fiber sensing Technology, Ed. Wiley \& Sons, 2002.

P. Russel, "Photonic Crystal Fibers", Science, Vol. 299, No. 17 January, pp. 358-362 (2003)

John M Fini, "Microstructure fibres for optical sensing in gases and liquids", Meas. Sci. Technol. 15, 1120-1128 (2004).

T. Ritari, J. Tuominen, H. Ludvigsen, J.C. Petersen, T. Sorensen, T.P. Hansen, H.R. Simonsen, “Gas sensing using air-guiding photonic bandgap fibers," Opt. Express 12, 4080-4087 (2004).

5 L.W. Kornaszewski, N. Gayraud, J.M. Stone, W.N. MacPherson, A.K. George, J.C. Knight, D.P. Hand, D.T. Reid, "Mid-infrared methane detection in a photonic bandgap fiber using a broadband optical parametric oscillator," Opt. Express 15, 11219-11224 (2007).

6 A.M. Cubillas, J.M. Lazaro, M. Silva-Lopez, O.M. Conde, M. Petrovich, J.M. Lopez-Higuera, High sensitive methane sensor based on a photonic bandgap fiber, EWOFS'07 (2007).

7 J.C. Knight, J. Broeng, T.A. Birks and P. St. J. Russell, "Photonic band gap guidance in optical fibers", Science, 282, 1476-8 (1998).

8 Y.L. Hoo, W. Jin, H.L. Ho, J. Ju, D.N. Wang, "Gas diffusion measurement using hollow-core photonic bandgap fiber", Sensors and Actuators B 105, 183-186, (2005).

9 H. Lehmann, S. Bruckner, J. Kobelke, G. Schwotzer, K. Schuster, R. Willsch,"Toward photonic crystal fiber based sensor distributed chemosensors", Proc. of SPIE 5855, 421, (2005).

10 C. J. Hensley, D. H. Broaddus, C. B. Schaffer, and A. L. Gaeta, "Photonic band-gap fiber gas cell fabricated using femtosecond micromachining," Opt. Express 15, 6690-6695 (2007).

11 A. van Brakel, C. Grivas, M.N. Petrovich, and D.J. Richardson, "Micro-channels machined in microstructured optical fibers by femtosecond laser," Opt. Express 15, 8731-8736 (2007)

12 Y. L. Hoo, W. Jin, C. H. L. Ho, D. N. Wang, S. C. Ruan, "Design and modelling of a photonic crystal fiber gas sensor", Applied Optics 42, 3509 -3515 (2003).

13 J. P. Carvalho, F. Magalhães, O. V. Ivanov, O. Frazão, F. M. Araújo, L. A. Ferreira, J. L. Santos, "Evaluation of coupling losses in hollow-core photonic crystal fibres", Proc. of SPIE 6619, (2007) 